\title{
Molecular Marker Characterization of Commercially Used Cytoplasmic Male Sterilities in Onion
}

\author{
Michael J. Havey \\ Department of Horticulture, University of Wisconsin, 1575 Linden Drive, Madison, WI 53706 \\ Sunggil Kim \\ Department of Horticulture, Biotechnology Research Institute, Chonnam National University, \\ Gwangju 61186, Republic of Korea
}

\begin{abstract}
Additional INDEX words. Allium cepa, CMS, cytoplasmic diversity, hybrid seed production
Abstract. Hybrid-onion (Allium cepa) seed is produced using systems of cytoplasmic male sterility (CMS) and two different CMS systems have been genetically characterized. $S$ cytoplasm was the first source of onion CMS identified in the 1920s, followed by $T$ cytoplasm that was described in the 1960s. Numerous studies have documented polymorphisms in the organellar DNAs differentiating $S$ and $T$ cytoplasms from the normal male-fertile cytoplasm of onion. There may be additional source(s) of onion CMS that have been described as "T-like" and appear to be more similar to $\mathrm{N}$ and $\mathrm{T}$ cytoplasms than $\mathrm{S}$ cytoplasm. In this study, onion breeding lines from commercial entities were evaluated for molecular markers distinguishing sources of onion CMS. Our results reveal that bona fide $T$ cytoplasm is rarely used commercially to produce hybrid-onion seed, and both $S$ cytoplasm and "T-like" cytoplasm are widely used. We propose that this "T-like" cytoplasm be labeled as " $R$ " cytoplasm because it may have originated from population(s) of 'Rijnsburger' onion in the Netherlands. The results of this study also help to clarify inconsistent reports regarding nuclear male-fertility restoration for different sources of onion CMS.
\end{abstract}

Hybrid-onion (Allium cepa) seed is produced using sources of CMS. The first source of onion CMS was described by Jones and Clarke (1943), and male sterility is conditioned by the interaction of the male-sterile (S) cytoplasm with the homozygous recessive genotype at one nuclear male-fertility restoration $(M s)$ locus. A dominant allele at $M s$ conditions male fertility for plants possessing S cytoplasm. Jones and Davis (1944) recognized that male-sterile plants ( $\mathrm{S}$ msms) can be seed propagated by crossing with male-fertile plants with normal $(\mathrm{N})$ male-fertile cytoplasm and homozygous recessive at $M s$ ( $N$ msms), referred to as the maintainer line. S cytoplasm has been commonly used to produce hybrid-onion seed since release of the first male-sterile inbred lines in 1952 (Goldman et al., 2000). Molecular analyses of the organellar genomes of $\mathrm{N}$ and $\mathrm{S}$ cytoplasms of onion have revealed many polymorphisms in both the chloroplast (cp) and mitochondrial (mt) DNAs (de Courcel et al., 1989; Havey, 1993; Holford et al., 1991; Kim et al., 2009; Sato, 1998; Satoh et al., 1993; von Kohn et al., 2013), likely because S cytoplasm is an alien cytoplasm introgressed from another Allium species into onion potentially via the triploid top-setting onion "Pran" (Havey, 1993). Another possibility is that S and N cytoplasms existed as sympatric cytoplasmic variants in the progenitor of onion, and were maintained during domestication and dispersal of onion from its center of origin in Central Asia throughout the world.

$\mathrm{T}$ cytoplasm is a second source of onion CMS that has been genetically characterized (Berninger, 1965), and male sterility is

Received for publication 6 May 2021. Accepted for publication 11 June 2021. Published online 22 July 2021.

We gratefully acknowledge the financial support of the U.S. Department of Agriculture (USDA), Agricultural Research Service (ARS), and the technical assistance of Christy Stewart of USDA-ARS.

M.J.H. is the corresponding author. E-mail: mjhavey@wisc.edu.

This is an open access article distributed under the CC BY-NC-ND license (https://creativecommons.org/licenses/by-nc-nd/4.0/). conditioned by the interaction of $\mathrm{T}$ cytoplasm with three malefertility restoration loci (Schweisguth, 1973). T cytoplasm has been used commercially to produce hybrid-onion seed, although its use is less common than S cytoplasm (Havey, 1994). Analyses of the cp and mt DNAs of $\mathrm{N}$ and $\mathrm{T}$ cytoplasms revealed few polymorphisms, indicating a close relationship between these two cytoplasms (de Courcel et al., 1989; Havey, 2000; Holford et al., 1991; Kim et al., 2019b). De Courcel et al. (1989) proposed that $\mathrm{N}$ and $\mathrm{T}$ cytoplasms arose from a progenitor (M) cytoplasm, and $\mathrm{T}$ cytoplasm is a relatively recent cytoplasmic variant closely related to $\mathrm{N}$ cytoplasm of onion.

There may be additional sources of CMS independently isolated and used to produce onion hybrids. Havey (2000) reported that sources of CMS used in Japan and the Netherlands were not $\mathrm{S}$ cytoplasmic and possessed mitochondrial polymorphisms differing from both $\mathrm{N}$ and T cytoplasms. Kim and Kim (2019) and Kim et al. (2009, 2019a, 2019b) reported variants of T cytoplasm (identified as "T," "T-like," and "Y") that showed differential stoichiometry of the mitochondrial orf725 and coxI genes. These studies indicate that there may be additional sources of onion CMS that are related, but not identical, to the T cytoplasm genetically characterized by Berninger (1965).

There are also conflicting results regarding the genetics of male-fertility restoration for the different sources of onion CMS. Numerous studies support male-fertility restoration of S cytoplasm by the $M s$ locus (Gökçe et al., 2002; Havey, 2013; Jones and Clarke, 1943; Kim et al., 2015a; Melgar and Havey, 2010). Kim et al. (2015a) proposed AcPMS1 as the candidate gene for $M s$, and segregation of this marker and the tightly linked jnurf13 marker (Kim, 2014) were consistent with male-fertility restoration of S cytoplasmic plants from North American (Havey and von Kohn, 2017) and Asian (Kim et al., 2015a) populations, but not necessarily for onion populations from the Indian subcontinent (Khar and Saini, 2016). Havey (2000) crossed the dominant restoration allele at $M s$ onto $\mathrm{T}$ cytoplasmic male-sterile plants 
and observed no restoration of male fertility. Kim (2014) observed male-fertility restoration of a "T-like" CMS by the dominant $M s$ allele, and proposed that the dominant allele at $M s$ restores male fertility for both $\mathrm{S}$ and $\mathrm{T}$ cytoplasms. De Vries and Wietsma (1992) crossed a CMS line from 'Rijnsburger' onion with the wild species Allium roylei as the male and reported male fertile progenies. These seemingly contradictory results could be explained if there were numerous CMS systems in onion, in addition to the genetically characterized $\mathrm{S}$ and $\mathrm{T}$ cytoplasms.

Table 1. Proposed classification of onion cytoplasms as N, R, S, or T as determined by polymorphisms in the chloroplast (cp) or mitochondrial (mt) DNAs from commercial and control inbred lines of onion, as well as predicted genotypes at the nuclear $M s$ locus as determined by the AcPMS1 marker.

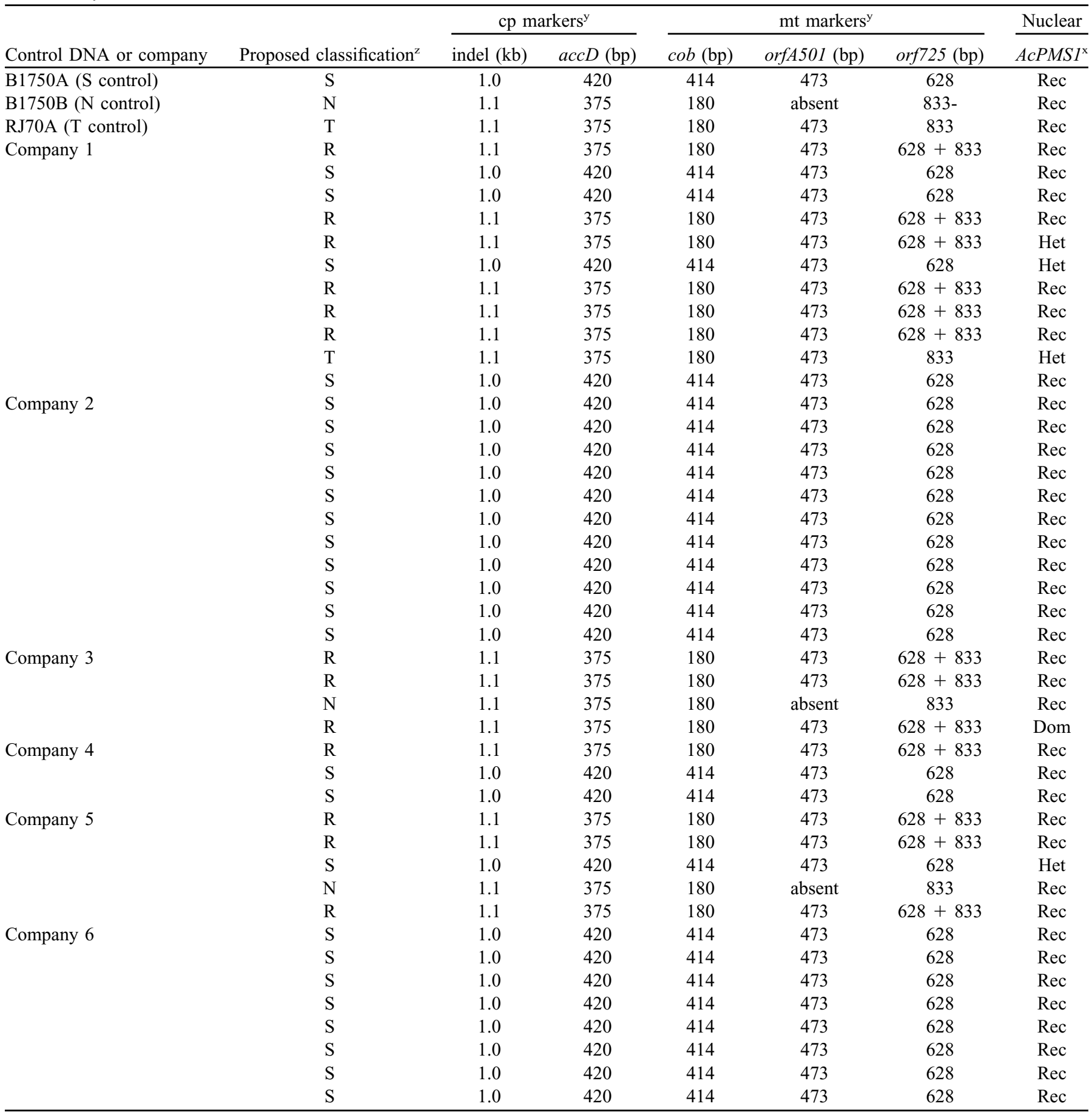

${ }^{\mathrm{z}}$ Normal (N) male-fertile and male-sterile (S) cytoplasms as described by Jones and Clarke (1943); male-sterile (T) cytoplasm as described by Berninger (1965); and a novel male-sterile (R) cytoplasm putatively selected from "Rijnsburger" onion.

${ }^{\mathrm{y}}$ Marker states in kilobases $(\mathrm{kb})$ or base pairs (bp) for various cytoplasms are presented Table 2: cp indel (Havey, 1995), cp accD (von Kohn et al., 2013), mt cob (Engelke et al., 2003), mt orfA501 (Engelke et al., 2003), and mt orf725 (Kim et al., 2009).

${ }^{\mathrm{x}}$ Genotypes at $M S$ are $\mathrm{Rec}=$ homozygous recessive; Het $=$ heterozygous; and Dom = homozygous dominant (Kim et al., 2015a). 
Given the uncertainties with regards to the diversity of onion CMS systems and the genetics of nuclear male-fertility restoration, we undertook this study to genotype polymorphisms in the organellar DNAs across commercially used sources of CMS to estimate their diversity and prevalence, before attempting to work out the genetics of different male-fertility restoration systems. Our results reveal that $\mathrm{S}$ cytoplasm is commonly used to produce hybrid-onion seed, as well as another source of CMS that may have originated from 'Rijnsburger' onion in the Netherlands. Bona fide $\mathrm{T}$ cytoplasm appears to be rarely used for production of hybrid-onion seed.

\section{Materials and Methods}

Seeds were voluntarily donated by private companies of proprietary inbred lines used for commercial production of hybridonion cultivars (Table 1). Origins of inbred lines with $\mathrm{N}$ (B1750B), S (B1750A), or T (RJ70A) cytoplasm were previously described by Havey (2000). About 50 seeds of each onion line were planted in a greenhouse in a soilless mix (PRO-MIX HP Mycorrhizae; Premier Tech Horticulture, Quakertown, PA) in 11.4-cm pots and plants were grown in a greenhouse at $28^{\circ} \mathrm{C}$ days and $25^{\circ} \mathrm{C}$ nights with supplemental lighting by sodium vapor lamps for $14 \mathrm{~h}$ per day. Four weeks after planting, leaf tissue was harvested in bulk from seedlings of each onion line, frozen in liquid $\mathrm{N}_{2}$, and freeze-dried. Tissue was then pulverized and DNA isolated using a kit (NucleoSpin Plant II Midi; Macherey-Nagel, Düren, Germany) according to the manufacturer's instructions. DNA concentrations were quantified with a spectrophotometer (NanoDrop ND-1000; Thermo Fisher Scientific, Waltham, MA) and quality was assessed by electrophoresis of DNA through 1\% agarose gels. Molecular markers distinguishing onion cytoplasms were indel markers in an intergenic region (Havey, 1995) and the $a c c D$ gene (von Kohn et al., 2013) in the cp DNA that distinguish $\mathrm{S}$ cytoplasm from $\mathrm{N}$ and $\mathrm{T}$ cytoplasms; the $c o b$ and orf $A 501$ polymorphisms in the mt DNA that distinguish N, S, and T cytoplasms (Engelke et al., 2003); and the orf725 marker in the mt DNA that distinguishes $\mathrm{N}, \mathrm{S}$, and T cytoplasms (Kim et al., 2009). The DNAs were also genotyped for the nuclear AcPMS1 marker that distinguishes alleles at the $M s$ locus (Kim et al., 2015a).

\section{Results and Discussion}

Markers in the cp and mt DNAs clearly distinguished S cytoplasm from the other onion cytoplasms (Table 1), in agreement with previous studies (de Courcel et al., 1989; Holford et al., 1991; Havey, 1993; Kim et al., 2015b; Sato, 1998; Satoh et al.,

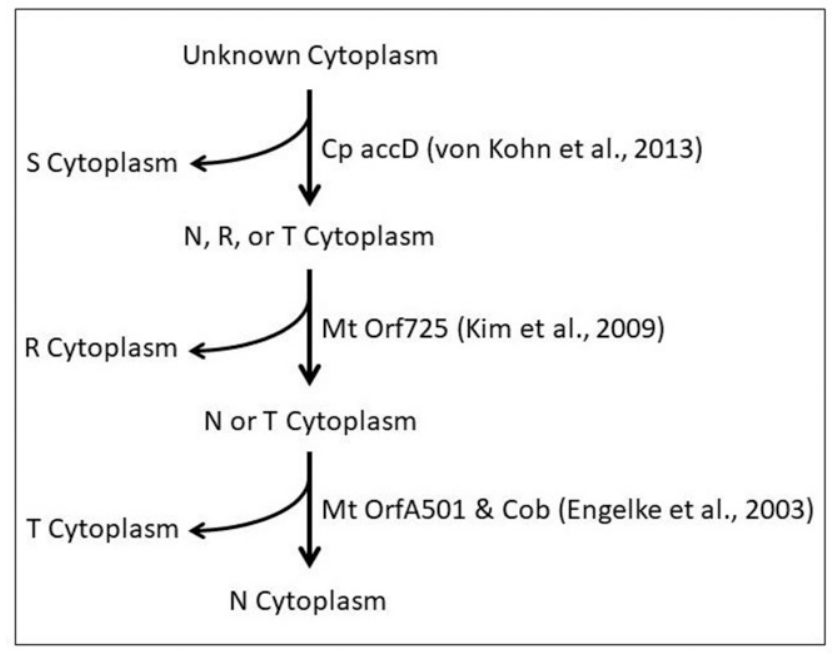

Fig. 1. Molecular markers distinguishing onion cytoplasms.

1993; von Kohn et al., 2013). We recommend that markers in the cp DNA be used first to separate $\mathrm{S}$ cytoplasm from other onion cytoplasms (Fig. 1; Table 2). All but two of the commercial lines with $\mathrm{S}$ cytoplasm were homozygous recessive at $M s$ as determined by the AcPMS1 marker (Kim et al., 2015a), as expected if these lines are used as female parents for production of hybrid seed. However, two of the commercial lines with $\mathrm{S}$ cytoplasm were scored as heterozygous at $M s$ and warrant further study. Melgar and Havey (2010) reported that the dominant allele at Ms can show reduced penetrance, complicating efforts to purge dominant alleles from an inbred line or population. Two of the commercial lines were classified as N-cytoplasmic across all cp and mt markers (Table 1), homozygous recessive at $M s$ based on the AcPMS1 marker, and therefore are likely maintainer lines.

The $c o b$ and orfA501 markers in the mt DNA developed by Engelke et al. (2003) correctly classified S, N, and bona fide T cytoplasms (inbred lines B1750A, B1750B, and RJ70A, respectively; detailed in Table 1). Only one commercially used CMS line from company \#1 (Table 1) had T cytoplasm, indicating that this cytoplasm is rarely used to produce hybrid-onion seed. RJ70A, the T cytoplasm control (Table 1), was scored by the AcPMS1 marker as homozygous recessive at $M s$, agreeing with Havey (2000) who reported RJ70B (the maintainer line of RJ70A) did not restore male-fertility for S cytoplasmic malesterile lines. The commercially used source of T cytoplasm from company \#1 (Table 1) was scored by the AcPMS1 marker as heterozygous at $M s$, further supporting the report that $M s$ does not restore male-fertility for T cytoplasm (Havey, 2000).

Table 2. Polymorphic markers in the chloroplast (cp) and mitochondrial (mt) DNAs distinguishing onion cytoplasms based on the results of this study.

\begin{tabular}{|c|c|c|c|c|c|}
\hline \multirow[b]{2}{*}{ Marker } & \multirow[b]{2}{*}{ Reference } & \multicolumn{4}{|c|}{ Cytoplasms ${ }^{\mathrm{z}}$} \\
\hline & & $\mathrm{N}$ & $\mathrm{R}$ & $\mathrm{S}$ & $\mathrm{T}$ \\
\hline $\mathrm{cp}$ indel & Havey (1995) & 1.1-kb amplicon & 1.1-kb amplicon & 1.0-kb amplicon & 1.1-kb amplicon \\
\hline$c p$ accD & von Kohn et al. (2013) & 375-bp amplicon & 375-bp amplicon & 420-bp amplicon & 375-bp amplicon \\
\hline $\mathrm{mt} c o b$ & Engelke et al. (2003) & 180-bp amplicon & 180-bp amplicon & 414-bp amplicon & 180-bp amplicon \\
\hline mt orfA501 & Engelke et al. (2003) & Absent & Absent & 473-bp amplicon & 473-bp amplicon \\
\hline mt orf725 & Kim et al. (2009) & 833-bp amplicon & 628- and 833-bp amplicons & 628-bp amplicon & 833-bp amplicon \\
\hline
\end{tabular}

${ }_{\mathrm{z}}$ Normal (N) male-fertile cytoplasm and male-sterile cytoplasms S (Jones and Clarke, 1943) and T (Berninger, 1965). Male-sterile R cytoplasm was identified in this study. Sizes of amplicons in kilobases (kb) or base pairs (bp). 
Bona fide $\mathrm{T}$ (RJ70A) and N (B1750B) cytoplasms were not differentiated by the mitochondrial orf 725 marker (Kim et al., 2009) and both cytoplasms produced the 833-base pair (bp) amplicon (Table 1). All lines with $\mathrm{S}$ cytoplasm produced the 628-bp amplicon (Table 1). Another cytoplasm can be distinguished from N, S, and T by production of both the 628- and 833-bp amplicons from orf725 (Tables 1 and 2). This unique cytoplasm is more similar to $\mathrm{N}$ and $\mathrm{T}$ cytoplasms than $\mathrm{S}$ cytoplasm, and is widely used commercially (13 of the 42 commercial lines in Table 1). This unique source of CMS may trace back to 'Rijnsburger', an onion population commonly grown in the Netherlands. Banga and Petiet (1958) reported that they were able to select CMS and maintainer lines directly from the 'Primeur' strain of 'Rijnsburger' onion. Van der Meer and van Bennekom (1971) also reported that male-sterile and maintainer lines could be extracted from different strains of 'Rijnsburger' onion. At the time this was important because selection of CMS and maintainer lines directly from commercially grown populations avoided time-consuming backcrosses to introgress $\mathrm{S}$ cytoplasm, resulting in faster development of locally adapted hybrids. We recommend that the unique cytoplasm that produces both the 628- and 833-bp amplicons of orf725 (Kim et al., 2009) can be designated as " $R$ " cytoplasm because it may have been selected directly from 'Rijnsburger' onion. Most of the companies that provided CMS lines classified as R were from Europe or had European subsidiaries. The existence of R cytoplasm may explain the observation by Kim (2014) that the dominant allele at $M s$ restores male fertility for $\mathrm{T}$ cytoplasm, which disagrees with Havey (2000) who observed that the dominant allele at Ms did not restore male fertility in $\mathrm{T}$ cytoplasm. This discrepancy can be reconciled given that both the $\mathrm{R}$ and $\mathrm{S}$ cytoplasms produce 628-bp orf725 amplicons and male fertility is restored by the dominant $M s$ allele for both cytoplasms; bona fide T cytoplasm does not produce the 628-bp orf725 amplicon and is not restored by Ms (Havey, 2000).

In summary, this study reveals that three commercially used sources of onion CMS can be distinguished by markers in the cp and mt DNAs (Fig. 1; Table 2). S and R cytoplasms are commonly used sources of onion CMS; T cytoplasm as described by Berninger (1965) is rarely used commercially. S cytoplasm can be distinguished from $\mathrm{N}, \mathrm{R}$, and $\mathrm{T}$ cytoplasms by chloroplast markers (Havey, 1995; Kim et al., 2015b; von Kohn et al., 2013). $\mathrm{N}$ and $\mathrm{T}$ cytoplasms can be distinguished by mitochondrial polymorphisms $c o b$ and orfA501 developed by Engelke et al., (2003). R cytoplasm can be distinguished from the other cytoplasms by the presence of both 628- and 833-bp amplicons of mitochondrial orf725 (Kim et al., 2009). With these cytoplasmic markers, onion breeders should be able to confidently determine cytoplasms presently in commercial use.

Although we did not score male fertility vs. sterility across the onion inbreeds used in this study, genotypes at $M s$ as predicted by the AcPMS1 marker (Kim et al., 2015a) were consistent with previous reports of male-fertility restoration by dominant allele(s) at the $M s$ locus for S (Havey, 2000; Jones and Clarke, 1943) and R (Kim, 2014) cytoplasms, both of which produce the orf725 amplicon (Table 1). Dominant alleles at Ms do not condition male-fertility restoration for T cytoplasm (Havey, 2000), which does not produce the orf725 amplicon (Table 1). The classification of onion CMS systems proposed by this research will be helpful to design genetic studies of nuclear male-fertility restoration systems across commercially used male-sterile cytoplasms of onion.

\section{Literature Cited}

Banga, O. and J. Petiet. 1958. Breeding male sterile lines of Dutch onion varieties as a preliminary to the breeding of hybrid varieties. Euphytica 7:21-30.

Berninger, E. 1965. Contribution a l'etude de la sterilite male de l'oignon (Allium cepa L.). Ann. Amelior. Plant. 15:183-199.

de Courcel, A.G.L., F. Veder, and J.M. Boussac. 1989. DNA polymorphism in Allium cepa cytoplasms and its implications concerning the origin of onions. Theor. Appl. Genet. 77:793-798, doi: 10.1007/ BF00268328.

De Vries, J. and W. Wietsma. 1992. Allium roylei Stearn restores cytoplasmic male sterility of Rijnsburger onion ( $A$. серa L.). J. Genet. Breeding 46:379-382.

Engelke, T., D. Terefe, and T. Tatlioglu. 2003. A PCR-based marker system monitoring CMS-(S), CMS-(T) and (N)-cytoplasm in the onion (Allium cepa L.). Theor. Appl. Genet. 107:162-167, doi: 10.1007/s00122-003-1230-3.

Gökçe, A.F., J. McCallum, Y. Sato, and M.J. Havey. 2002. Molecular tagging of the $M s$ locus in onion. J. Amer. Soc. Hort. Sci. 127: 576-582, doi: 10.21273/JASHS.127.4.576.

Goldman, I.L., G. Schroeck, and M.J. Havey. 2000. History of public onion breeding programs and pedigree of public onion germplasm releases in the United States. Plant Breeding Rev. 20:67-103.

Havey, M.J. 1993. A putative donor of S cytoplasm and its distribution among open pollinated populations of onion. Theor. Appl. Genet. 86:128-134, doi: 10.1007/BF00223817.

Havey, M.J. 1994. The cytoplasms of sterile lines used to produce commercial hybrid-onion seed. Allium Improvement Nwsl. 4:25-27.

Havey, M.J. 1995. Identification of cytoplasms using the polymerase chain reaction to aid in the extraction of maintainer lines from open pollinated populations of onion. Theor. Appl. Genet. 90:263-268, doi: 10.1007/BF00222212.

Havey, M.J. 2000. Diversity among male sterility inducing and male fertile cytoplasms of onion. Theor. Appl. Genet. 101:778-782, doi: 10.1007/s001220051543.

Havey, M.J. 2013. Single nucleotide polymorphisms in linkage disequilibrium with the male-fertility restoration $(M s)$ locus of onion. J. Amer. Soc. Hort. Sci. 138:306-309, doi: 10.21273/JASHS.138. 4.306 .

Havey, M.J. and C. von Kohn. 2017. Efficacy of molecular markers jnurf13 and AcPms1 for prediction of genotypes at the nuclear Ms locus in North American open pollinated populations of onion. HortScience 52:1052-1053, doi: 10.21273/HORTSCI11954-17.

Holford, P., J.H. Croft, and H.J. Newbury. 1991. Differences between, and possible origins of, the cytoplasms found in fertile and male-sterile onions (Allium cepa L.). Theor. Appl. Genet. 82:737-744, doi: 10.1007/BF00227319.

Jones, H.A. and A. Clarke. 1943. Inheritance of male sterility in the onion and the production of hybrid seed. Proc. Amer. Soc. Hort. Sci. 43:189-194.

Jones, H.A., G. Davis. 1944. Inbreeding and heterosis and their relation to the development of new varieties of onions. U.S. Dept. Agr. Tech. Bul. 874.

Khar, A. and N. Saini. 2016. Limitations of PCR based molecular markers to identify male sterile and maintainer plants from Indian onion (Allium cepa L.) populations. Plant Breeding 135:519-524, doi: $10.1111 /$ pbr.12373.

Kim, B. and S. Kim. 2019. Identification of a variant of CMS-T cytoplasm and development of high resolution melting markers for distinguishing cytoplasm types and genotyping a restorer-of-fertility locus in onion (Allium cepa L.). Euphytica 215:164, doi: 10.1007/ s10681-019-2492-4. 
Kim, B., C. Kim, and S. Kim. 2019a. Inheritance of fertility restoration of male-sterility conferred by cytotype $\mathrm{Y}$ and identification of instability of male fertility phenotypes in onion (Allium cepa L.). J. Hort. Sci. Biotechnol. 94:341-348, doi: 10.1080/14620316.2018.1512383.

Kim, B., T. Yang, and S. Kim. 2019b. Identification of a gene responsible for cytoplasmic male-sterility in onions (Allium cepa L.) using comparative analysis of mitochondrial genome sequences of two recently diverged cytoplasms. Theor. Appl. Genet. 132:313-322, doi: 10.1007/s00122-018-3218-z.

Kim, S. 2014. A codominant molecular marker in linkage disequilibrium with a restorer-of-fertility gene (Ms) and its application in reevaluation of inheritance of fertility restoration in onions. Mol. Breed. 34:769-778, doi: 10.1007/s11032-014-0073-8.

Kim, S., C. Kim, M. Park, and D. Choi. 2015a. Identification of candidate genes associated with fertility restoration of cytoplasmic male sterility in onion (Allium cepa L.) using a combination of bulked segregant analysis and RNA seq. Theor. Appl. Genet. 128:2289-2299, doi: 10.1007/s00122-015-2584-z.

Kim, S., E. Lee, D.Y. Cho, T. Han, H. Bang, B.S. Patil, Y.K. Ahn, and M. Yoon. 2009. Identification of a novel chimeric gene, orf725, and its use in development of a molecular marker for distinguishing among three cytoplasm types in onion (Allium cepa $\mathrm{L}$.). Theor. Appl. Genet. 118:433-441, doi: 10.1007/s00122-008-0909-x.

Kim, S., J.Y. Park, and T. Yang. 2015b. Comparative analysis of the complete chloroplast genome sequences of a normal male-fertile cytoplasm and two different cytoplasms conferring cytoplasmic male sterility in onion (Allium cepa L.). J. Hort. Sci. Biotechnol. 90:459-468, doi: 10.1080/14620316.2015.11513210.

Melgar, S. and M.J. Havey. 2010. The dominant $M S$ allele in onion shows reduced penetrance. J. Amer. Soc. Hort. Sci. 135:49-52, doi: 10.21273/JASHS.135.1.49.

Sato, Y. 1998. PCR amplification of CMS-specific mitochondrial nucleotide sequences to identify cytoplasmic genotypes of onion (Allium cepa L.). Theor. Appl. Genet. 96:367-370, doi: 10.1007/ s001220050750.

Satoh, Y., M. Nagai, T. Mikami, and T. Kinoshita. 1993. The use of mitochondrial DNA polymorphism in the classification of individual onion plants by cytoplasmic genotypes. Theor. Appl. Genet. 86:345-348, doi: 10.1007/BF00222100.

Schweisguth, B. 1973. Etude d'un nouveau type de sterilite male chez l'oignon, Allium cepa L. Ann. Amelior. Plant. 23:221-233.

Van der Meer, Q.P. and J.L. van Bennekom. 1971. Frequencies of genetical factors determining male sterility in onion (Allium cepa) and their significance for the breeding of hybrids. Institute of Horticultural Plant Breeding. Wageningen, the Netherlands.

Von Kohn, C., A. Kiełkowska, and M.J. Havey. 2013. Sequencing and annotation of the chloroplast DNAs of normal $(\mathrm{N})$ male fertile and male sterile (S) cytoplasms of onion and single nucleotide polymorphisms distinguishing these cytoplasms. Genome 56:737-742, doi: 10.1139/gen-2013-0182. 